\title{
Análise do movimento vertical sob duas diferentes configurações de altos níveis da troposfera
}

Analysis of vertical movement in two different configurations of high-levels of the troposphere

Fernando Cossetin ${ }^{1}$, André Becker Nunes ${ }^{2}$ e Mateus da Silva Teixeira ${ }^{3}$

${ }^{1}$ Bacharel, Faculdade de Meteorologia, Universidade Federal de Pelotas, Pelotas, Brasil
${ }^{2}$ Prof. Dr., Faculdade de Meteorologia, Universidade Federal de Pelotas, Pelotas, Brasil
${ }^{3}$ Prof. Dr., Faculdade de Meteorologia, Universidade Federal de Pelotas, Pelotas, Brasil

\begin{abstract}
Resumo
A região Sul do Brasil é potencialmente favorável a ocorrência de eventos extremos, onde estes são decorrentes de diversos sistemas meteorológicos que atuam na América do Sul. Logo diversas técnicas foram expostas para melhorar a precisão dos mesmos e uma característica presente em muitas delas é a presença de cavados nos níveis de $500 \mathrm{hPa}$. Assim, o presente trabalho visa avaliar o movimento vertical de dois eventos de tempestade severa que atingiram a Região Sul do Brasil no outono de 2013, sendo uma no dia 26 de Março e outra no dia 29 de Maio, onde ambos decorreram do mesmo tipo de sistema porém com diferentes inclinações do eixo horizontal dos cavados nos altos níveis da atmosfera. Foram utilizados dados da compilação mensal de casos significativos do CPTEC/INPE para a escolha dos eventos de instabilidade severa. Para a elaboração dos campos meteorológicos a serem avaliados foram utilizados dados de reanálise MERRA. Este estudo mostrou que o movimento vertical ômega ascendente foi muito mais intenso quando relacionado com o sistema em superfície associado ao cavado negativamente inclinado (cerca de 1,2 $\mathrm{m} \mathrm{s}^{-1}$,) enquanto que o movimento ascendente associado ao cavado positivo fica em torno de $0,5 \mathrm{~m} \mathrm{~s}^{-1}$.
\end{abstract}

Palavras-chave: Inclinação do eixo horizontal dos cavados em altos níveis, tempestades severas, movimento ascendente.

\begin{abstract}
The southern region of Brazil is potentially favorable the occurrence of extreme events, where these are due to different weather systems that operate in South America. Therefore various techniques have been exposed to improve the accuracy thereof, a characteristic what is present in many of them is the presence of troughs in the $500 \mathrm{hPa}$ level. The present study aims to evaluate the vertical movement of two severe storm events that hit southern Brazil in the fall of 2013, one on 26 March and another on May 29, where both of them occurred from the same system type, but with different inclinations of the horizontal axis of the higher level trough of the atmosphere. For this were used data from the monthly compilation of significant cases of CPTEC / INPE for the choice of severe instability events. For the preparation of meteorological fields to be evaluated were reanalysis data used MERRA. This study showed that omega vertical upward movement was much more intense when related to the system associated with the negatively-trough (about $1.2 \mathrm{~m} \mathrm{~s}-1$ ) while the upward movement associated with the positive-trough was around $0.5 \mathrm{~ms}$-1.
\end{abstract}

Keywords: Slope the horizontal axis of the high-level troughs, severe storms, upward movement. 


\section{Introdução}

A região subtropical Sul-Americana situada a leste da Cordilheira dos Andes é dita como uma área favorável a ocorrência de eventos severos (FUJITA, 1973; VELASCO e FRITSCH, 1987; SILVA DIAS, 1999; NECHET, 2002; MARCELINO, 2003; BROOKS et al., 2003; NASCIMENTO, 2005; ZIPSER et al., 2006; SANCHEZ et al., 2008; entre outros). Dada as consequências de tais eventos à sociedade, o estudo dos fenômenos meteorológicos que decorrem em tais eventos possui cada vez mais importância. Assim diversos limiares de tempestades severas foram expostos pela literatura mundial, onde as mais aceitas são baseadas em granizo gigante (pedras de $2 \mathrm{~cm}$ ou mais de diâmetro ao atingir a superfície) e/ou rajadas de vento com força destrutiva (velocidade acima de $50 \mathrm{kt}$ ) (JOHNS; DOSWELL, 1992 e MOLLER, 2001) além de inundações repentinas e chuvas intensas (MILLS; COLQHOUN, 1998). Dentre os sistemas que atuam na América do Sul e afetam o tempo estão os sistemas frontais (ANDRADE, 2005), entre outros, tais como Sistemas Convectivos de Mesoescala, Vórtices Ciclônicos, etc.... A presença de cavados nos níveis superiores da atmosfera é uma característica associada a diversos sistemas meteorológicos Lefevre e Nielsen-Gammon (1995) apontam que o nível de $500 \mathrm{hPa}$ tem se mostrado ideal na identificação de cavados móveis na troposfera superior que afetam a troposfera inferior por meio de suporte dinâmico. Estudos elaborados para o Hemisfério Norte (MACDONALD, 1976; GLICKMAN et al., 1977; STRAHL; SMITH, 2001; GAZA; BOSART, 1990, HAKIM et al., 1995; DEAN et al., 1996; entre outros) indicam que cavados de altos níveis podem ser avaliados conforme a inclinação do seu eixo horizontal (podendo ser positiva, negativa ou nula), sendo que os com inclinação negativa estão associados a instabilidades extremamente severas.

Estudos relacionando esta inclinação do eixo horizontal dos cavados em altos níveis com instabilidades severas são muito mais frequentes no Hemisfério Norte. Star e Rosen
(1972) apontam que os movimentos ascendentes de escala sinótica, forçados principalmente por advecção de calor e momentum, são mais intensos para cavados com inclinação negativa.

Em análise aos invernos de 1972-72 e 1973-74 para a região dos Grandes Lagos (Estados Unidos da América), Glickman et al. (1977) evidenciaram através da Tabela 1 esta informação de que o movimento ascendente associado aos cavados com inclinação negativa do seu eixo horizontal é de maior magnitude.

Tabela 1: Movimento ascendente em unidades de 10-3 $\mathrm{mb} \mathrm{s}^{-1}$, região dos Grandes Lagos (EUA),

invernos de 1971-72 e 1973-74, relacionado a inclinação do cavado (GLICKMAN et al. (1977)).

\begin{tabular}{lll}
\hline & $\begin{array}{l}\text { Número } \\
\text { de casos }\end{array}$ & Média \\
\hline Todos os cavados & 696 & 2.77 \\
Inclinação positiva & 287 & 0.96 \\
Sem inclinação & 319 & 3.70 \\
Inclinação negativa & 90 & 5.53 \\
\hline
\end{tabular}

O presente trabalho visa avaliar o movimento vertical de dois eventos de tempestade severa ocorridos no outono de 2013 (um evento dia 23 de Março e outro dia 29 de Maio) no sul do Brasil decorrentes do mesmo tipo de sistema meteorológico em superfície associado a diferentes inclinações do eixo horizontal do cavado em altos níveis da atmosfera. Segundo compilação mensal de casos significativos elaborada pelo CPTEC/INPE (Centro de Previsão do Tempo e Estudos Climáticos / Instituto Nacional de Pesquisas Espaciais), tais eventos apresentaram significativa severidade onde no dia 23 de Março pelo menos 30 municípios registraram granizo o que acabou danificando telhados de construções, plantações na área rural e estufas, além de intensas rajadas de vento que ocasionaram em destelhamentos de casas. Enquanto que no evento do dia 29 de Maio foram registradas rajadas de mais de $100 \mathrm{~km} / \mathrm{h}$, 
destelhando diversas casas e derrubando postes da rede elétrica.

\section{Material e métodos}

\subsection{Dados utilizados}

Serão utilizados dados de reanálise MERRA (Modern Era Retrospective-analysis for Research and Applications) para a obtenção dos campos a serem avaliados, estes possuem resolução temporal de 3 horas, com espaçamento de grade de $0,66^{\circ}$ de longitude e $0,5^{\circ}$ de latitude, contabilizando assim uma grade horizontal com 540 pontos no eixo zonal e 361 pontos no eixo meridional, além de 42 níveis verticais $(1000 \mathrm{hPa}$ até $0,1 \mathrm{hPa})$, como descrito por Rienecker et al., (2011).

\subsection{Metodologia}

Serão analisados os campos de altura geopotencial em $500 \mathrm{hPa}$, velocidade vertical ômega em $500 \mathrm{hPa}$, linhas de corrente em 850 $\mathrm{hPa}$, umidade relativa em $850 \mathrm{hPa}$ e pressão ao nível médio do mar. Através destes será avaliada a relação entre os sistemas de alta e baixa troposfera.

Por fim, será identificado em qual caso os valores de ômega ascendente são mais intensos, visando validar parcialmente se o padrão identificado por Glickmann et. al. para o Hemisfério Norte é valido para o Hemisfério Sul.

\section{Resultados e discussão}

\subsection{Caso 1: Dia 26 de Março de 2013}

Analisando o dia que antecede a data do evento, nota-se a pré-existência de um cavado com inclinação positiva do seu eixo horizontal no nível de 500 hPa (Figura 1 (a)) associado ao avanço de um sistema frontal em superfície (Figura 2 (a)). Após a ocorrência do evento, este cavado em altos níveis se aprofunda tornandose um vórtice ciclônico (Figura 1 (c)) e devido a intensa advecção de vorticidade ciclônica corrente abaixo, decorre no processo de ciclogênese em superfície na costa da região $\begin{array}{llll}\text { sudeste do Brasil (Figura } 2 & \text { (c)). }\end{array}$

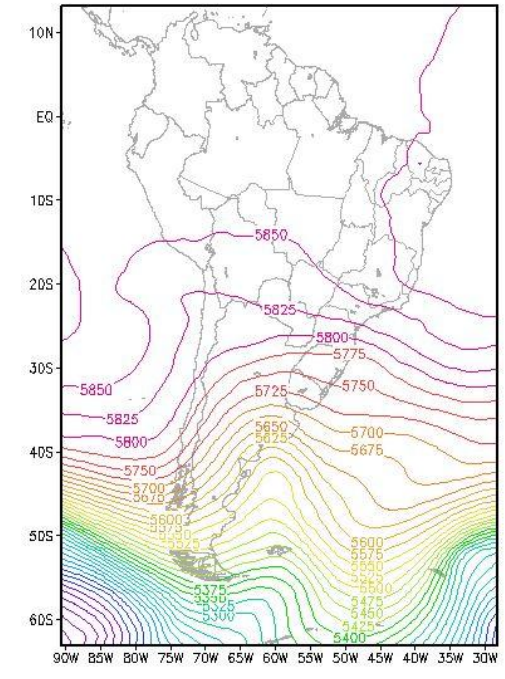

(a)

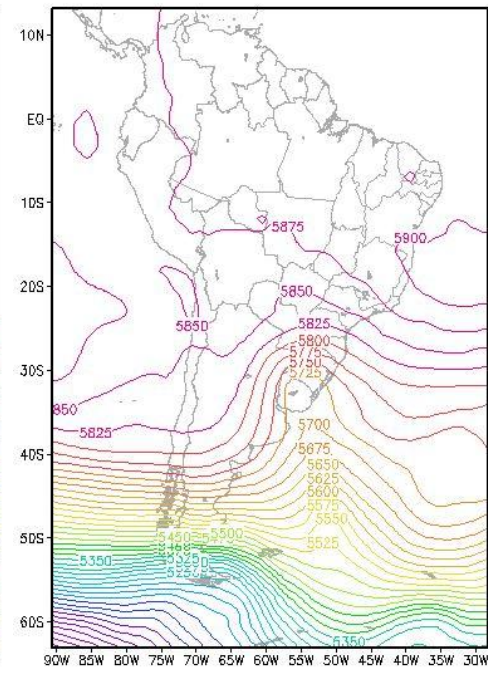

(b)

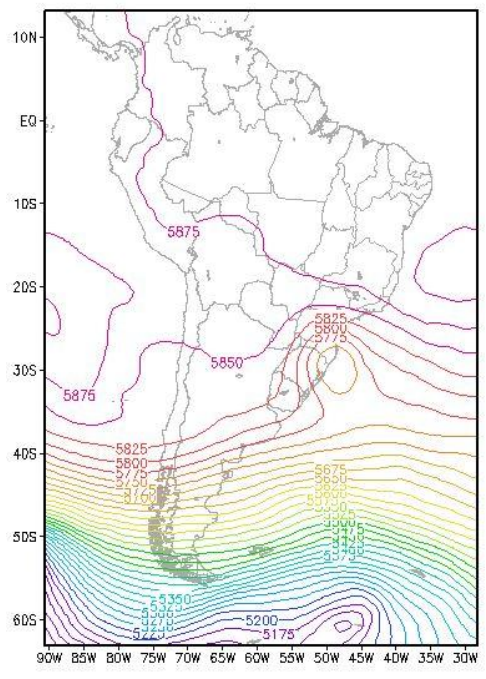

(c)

Figura 1. Altura geopotencial (mgp) no nível de 500 hPa às 12 UTC para (a) o dia anterior, (b) o dia da ocorrência e (c) um dia após a ocorrência do caso 1 de tempestade severa que atingiu a Região Sul do Brasil. 


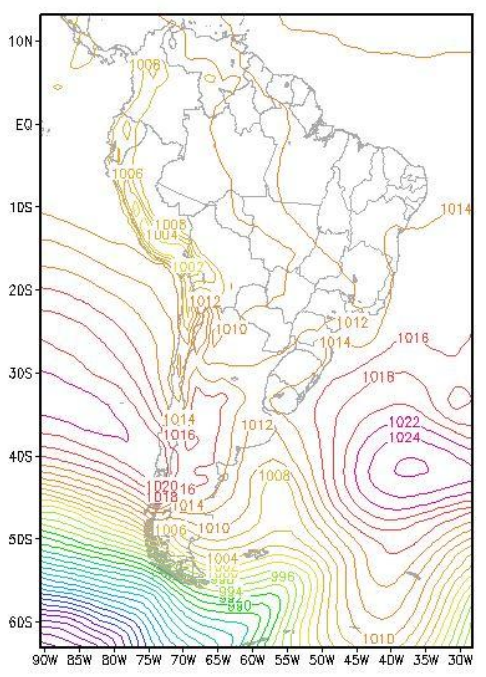

(a)

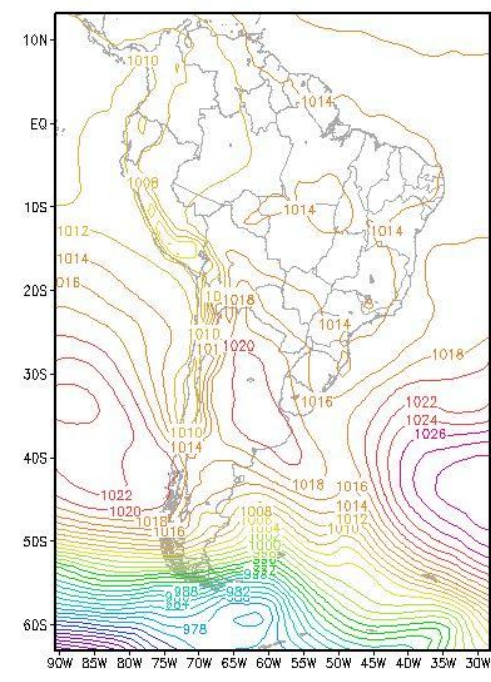

(b)

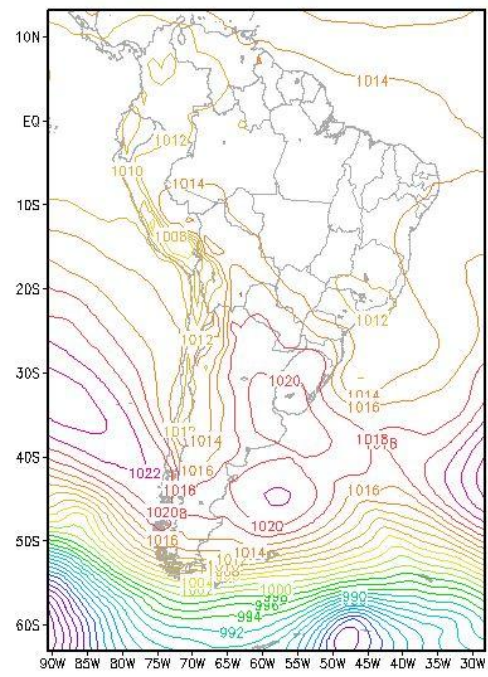

(c)

Figura 2. Pressão ao nível médio do mar (hPa) às 12 UTC para (a) o dia anterior, (b) o dia da ocorrência e (c) o dia posterior ao caso 1 de tempestade severa que atingiu a Região Sul do Brasil.

Com o aprofundamento do cavado positivamente inclinado a área com movimento

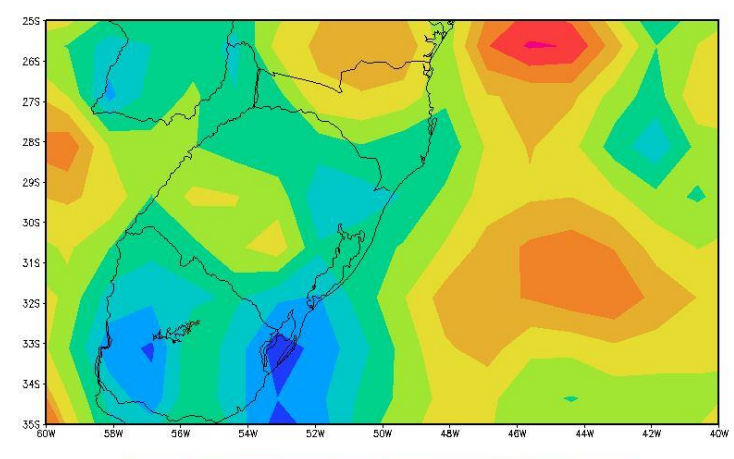

(a)

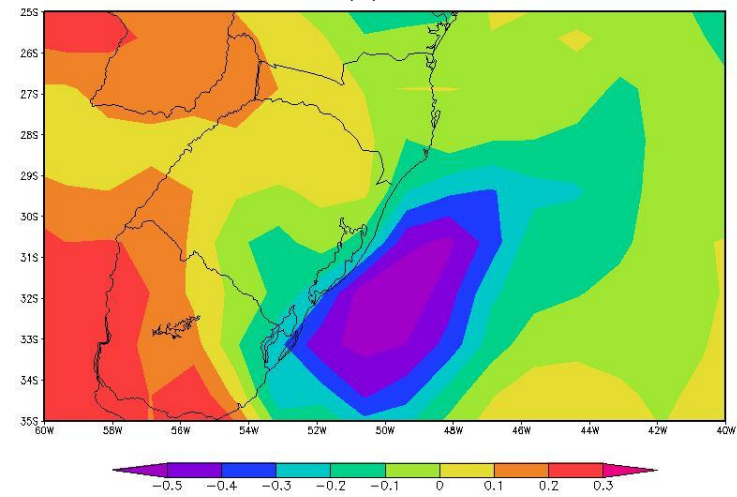

(c) vertical ascendente se intensifica e se amplia (Figuras 3 (a) a 3 (c)).

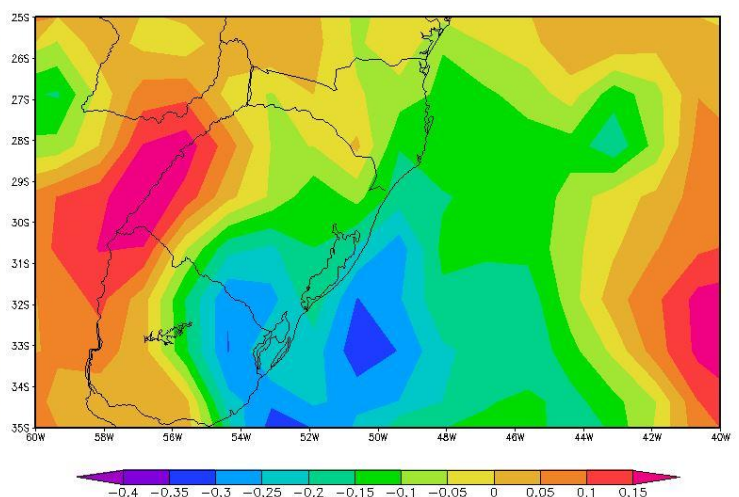

(b)

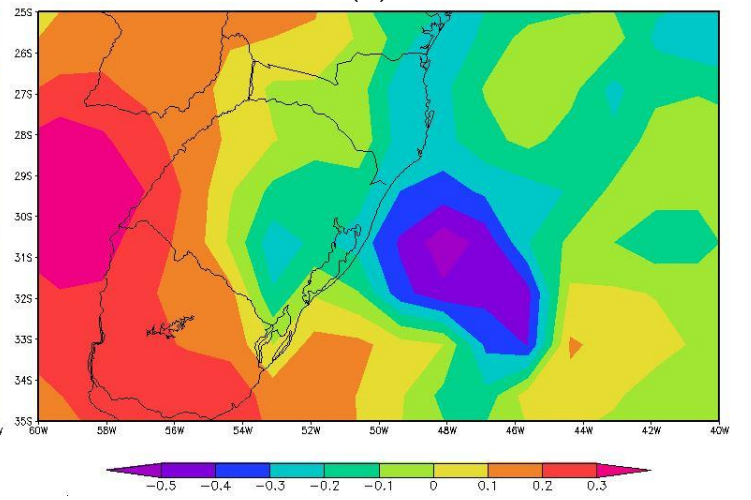

(d)

Figura 3. Movimento vertical ômega $\left(\mathrm{m} \mathrm{s}^{-1}\right)$ do dia do evento às (a) 00 UTC, (b) 06 UTC, (c) 12 UTC e (d) 18 UTC do dia do caso 1 de tempestade severa que atingiu a Região Sul do Brasil.

3.2 Caso 2: Dia 29 de Maio de 2013
Analisando o dia anterior a ocorrência do evento (Figura 4 (a)) nota-se a presença de um vórtice ciclônico prolongando um cavado 
com inclinação nula do seu eixo horizontal no nível de $500 \mathrm{hPa}$ em suporte ao avanço de um sistema frontal associado a um ciclone extratropical em superfície. Já durante o dia do evento de tempestade severa este vórtice desloca-se para leste e o cavado associado em altos níveis adquire leve inclinação positiva
(Figura 4 (b)). Para o dia após a ocorrência do evento este cavado torna-se negativamente inclinado (Figura 4 (c)), isto acaba por iniciar uma nova ciclogênese em superfície sobre o Oceano Atlântico a sudeste do RS (Figura 5 (c)).

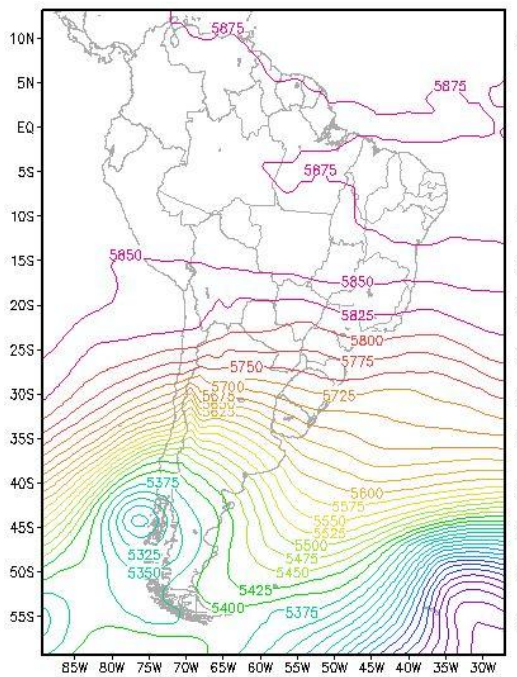

(a)

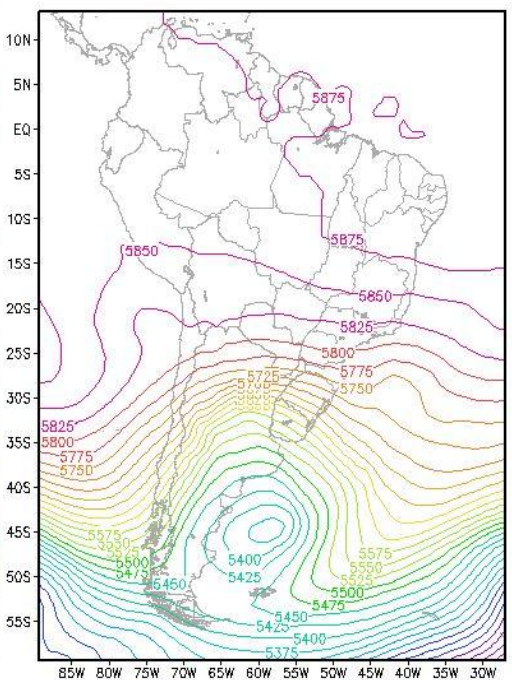

(b)

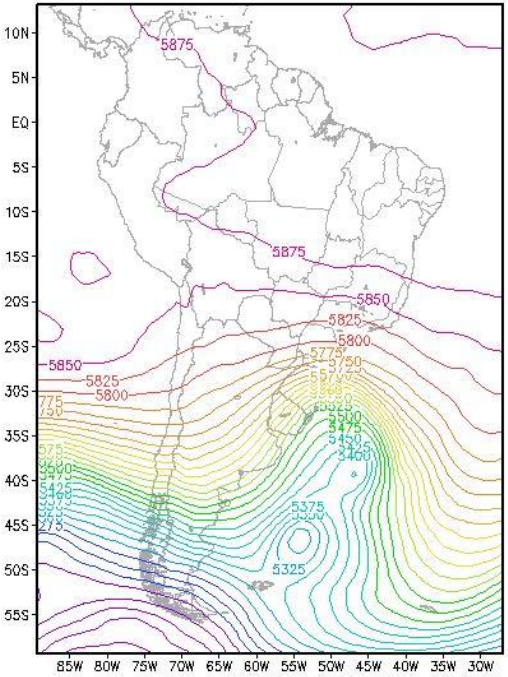

(c)

Figura 4. Altura geopotencial (mgp) no nível de 500 hPa às 12 UTC para (a) o dia anterior, (b) o dia da ocorrência e (c) um dia após a ocorrência do caso 2 de tempestade severa que atingiu a Região Sul do Brasil.

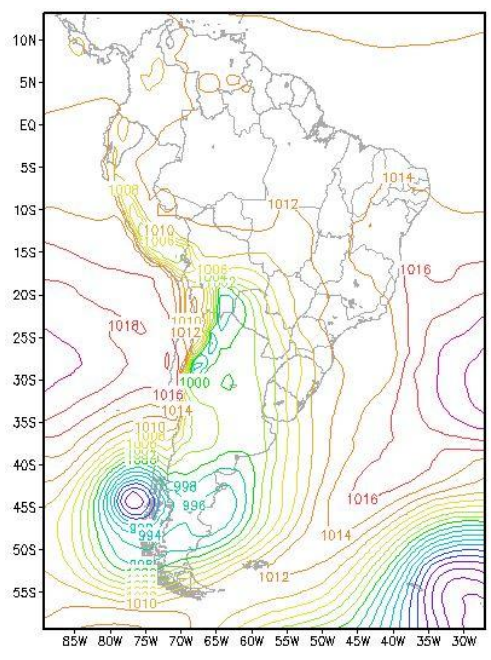

a)

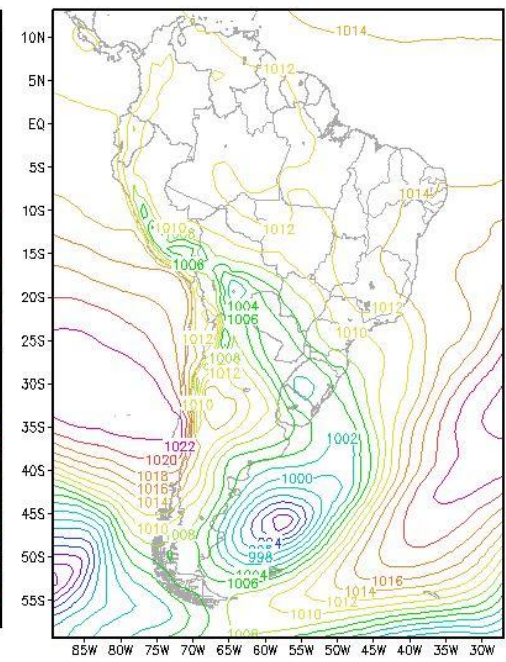

(b)

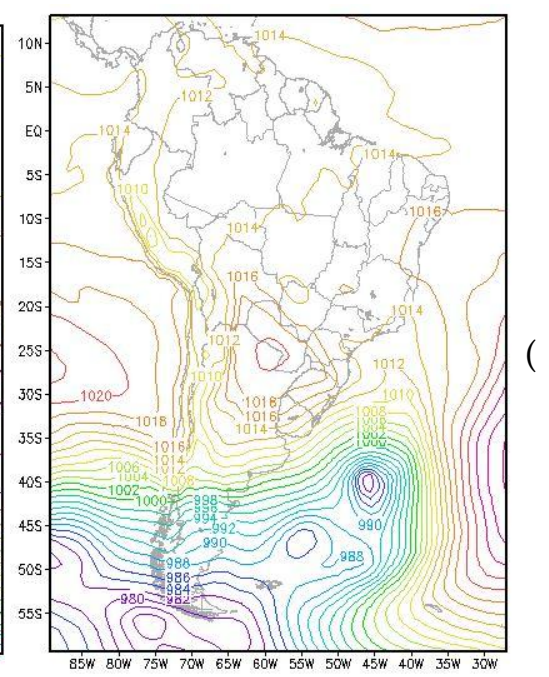

(c)

Figura 5. Pressão ao nível médio do mar (hPa) às 12 UTC para (a) o dia anterior, (b) o dia da ocorrência e (c) o dia posterior ao caso 2 de tempestade severa que atingiu a Região Sul do Brasil.

Analisando as figuras de velocidade vertical ômega nota-se intenso movimento ascendente relacionado ao sistema frontal associado ao primeiro ciclone extratropical (Figura 6 (a)), entretanto esta área perde intensidade ao decorrer do dia do caso 2 devido 
ao enfraquecimento do ciclone e da mudança da inclinação do cavado em altitude de positiva a negativa (Figuras 6 (b) e 6 (c)). O novo ciclone extratropical decorrente do cavado

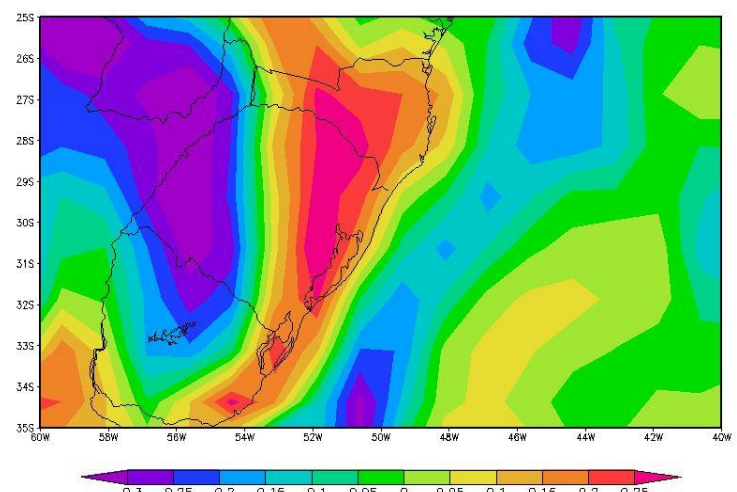

(a)

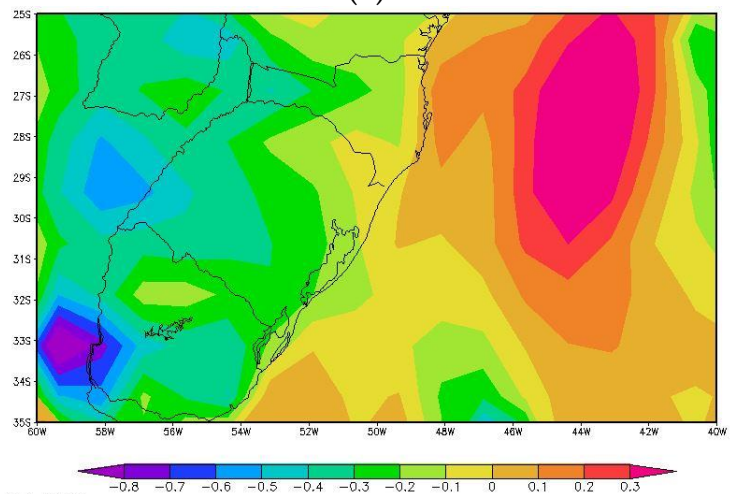

(c) negativamente inclinado decorre em uma pequena área com movimento ascendente extremamente intenso, assumindo valores de $\begin{array}{llllll}1,2 & \mathrm{~m} & \mathrm{~s}^{-1} & \text { (Figura } & 6 & \text { (d)). }\end{array}$

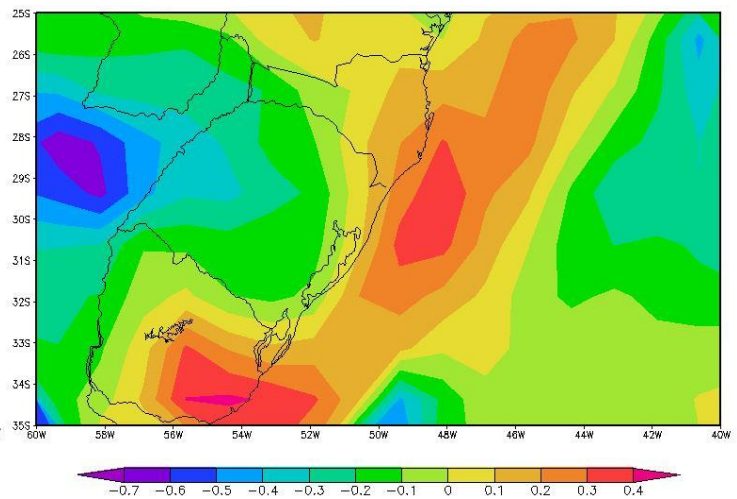

(b)

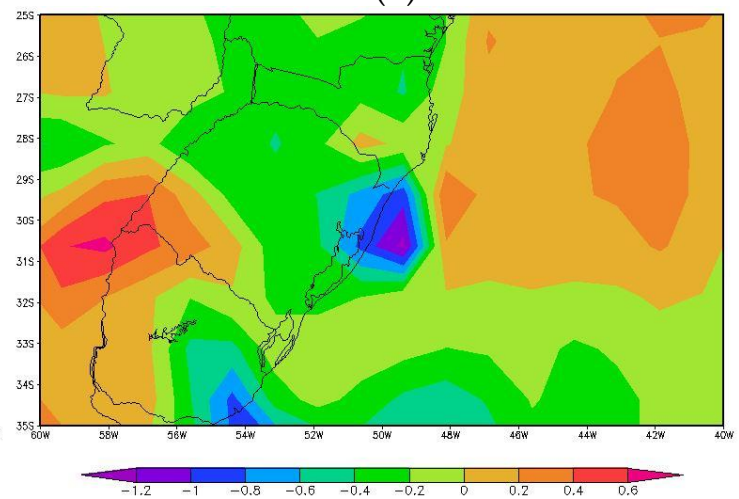

(d)

Figura 6. Movimento vertical omega $\left(\mathrm{m} \mathrm{s}^{-1}\right)$ do dia do evento às (a) 00 UTC, (b) 06 UTC, (c) 12 UTC e (d) 18 UTC do dia do caso 2 de tempestade severa que atingiu a Região Sul do Brasil.

\section{Conclusões}

O movimento vertical (ômega) ascendente foi muito mais intenso quando associado ao cavado com inclinação negativa do eixo horizontal em $500 \mathrm{hPa}$, cerca de 1,2 $\mathrm{m} \mathrm{s}^{-1}$, enquanto que o movimento ascendente relacionado ao evento associado ao cavado positivo apontou magnitude em cerca de $0,5 \mathrm{~m}$ $\mathrm{s}^{-1}$. Isto vai ao encontro do proposto por

Glickmann et al., (1977) em estudo a região dos Grandes Lagos nos Estados Unidos da América.

Além disto, o cavado com inclinação negativa produziu áreas de intensa instabilidade mais localizadas, enquanto que o positivamente inclinado esteve associado a áreas de instabilidade com menor magnitude e maior área de abrangência.

\section{Referências}

Andrade, K. M. Climatologia e comportamento dos sistemas frontais sobre a América do Sul. Climatologia e comportamento dos sistemas frontais sobre a América do Sul, 2005.

Brooks, H. E., Lee, J. W. and Craven, J. P. The spatial distribution of severe thunderstorm and tornado environments from global reanalysis data. Atmos. Research, v. 67-68, p. 73-94, 2003.

Dean, Devin B., Bosart, Lanec F. Northern Hemisphere $500-\mathrm{hPa}$ trough merger and fracture: A climatology and case study. 
Monthly weather review, v. 124, n. 12, p. 26442671, 1996.

Fujita, T. T. Tornadoes around the world. Weatherwise, v.26, p.56-62, 1973.

Gaza, R. S. and L. F. Bozart, 1990: Troughmerger characteristics over North America. Wea. Forecasting, 5, 314-331.

Glickman, Todd., MacDonald, Normal J., Sanders, Frederick. New Findings an the Apparent Relationship between Convective Activity and the Shape of $500 \mathrm{mb}$ Troughs. Monthly Weather Review, v. 105, p. 1060, 1977.

Hakim, G. J., Bosart, L. F. and D. Keyser, 1995: The Ohio Valley wave-merger cyclogenesis event of 25-26 January 1978. Part I: Multiscale case study. Mon. Wea. Rev., 123, 2663-2692.

Johns, R. H. and Doswell, C. A. Severe local storms forecasting. Wea. Forecasting, v. 7, p. 588-612, 1992.

Lefevre, R. J. and Nielsen-Gammon, R. J., 1995: An objective climatology of mobile troughs in the Northern Hemisphere. Tellus, 47A, 638655.

MacDonald, N. J. On the apparent relationship between convective activity and the shape of $500 \mathrm{mb}$ troughs. Monthly Weather Review, v. 104, n. 12, p. 1618-1622, 1976.

Marcelino, I, P. V. O. Análise de episódios de tornado em Santa Catarina: caracterização sinótica e mineração de dados. Dissertação (Mestrado em Sensoriamento Remoto) Instituto Nacional de Pesquisas Espaciais, São José dos Campos, São Paulo, 2003.

Mills, G. A., Colquhoun, J. R. Objective prediction of severe thunderstorm environments: preliminary results linking a decision tree with an operational regional NWP model. Wea. Forecasting, v. 13, p.10781092, 1998.

Moller, A. R. Severe local storms forecasting. In: Severe Convective Storms, C. A. Doswell III (Ed.), Amer. Meteor. Soc. Monograph v. 28, n. 50, p. 433-480, 2001.

Nascimento, E. L. Previsão de tempestades severas utilizando-se parâmetros convectivos e modelos de mesoescala: uma estratégia operacional adotável no Brasil. Revista Brasileira de Meteorologia, v. 20, n. 1, p. 121140, 2005.

Nechet, D. Ocorrência de tornados no Brasil. Bol. Soc. Bras. Meteor., v.26, p.29-39, 2002.

Rienecker, Michele M., et al. "MERRA: NASA's modern-era retrospective analysis for research and applications." Journal of Climate 24.14 (2011): 3624-3648.

Sánches, J. L. et al. Short-term forecast of thunderstorm in Argentina. Atmosf. Reserarch, v.88, p.36-45, 2008.

SILVA DIAS, M. A. F. Storms in Brazil, In: Hazzards and Disasters Series, Storms Volume II, R. Pielke Se. e R. Pielke Jr (Eds), p.207219, 1999.

Velasco, I, Fritsch, J. M. Mesoescale Convective Complexes in the Americas. J. Geph. Res., v.92, p.9591-9613, 1987.

Zipser, E. J., Cecil, D. K. J., Liu, C., Nesbitt, S. W., Yorty, D. P. Where are the most intense thunderstorms on earth? Bull. Amer. Meteor. Soc., v. 87, p.1057-1071, 2006. 